\title{
Induction and Reversal of Long-Term Potentiation by Low- and High-Intensity Theta Pattern Stimulation
}

\author{
Deborah S. Barr, ${ }^{1}$ Nevin A. Lambert, ${ }^{1,3}$ Kendall L. Hoyt, ${ }^{2,3}$ Scott D. Moore, ${ }^{2,3}$ and Wilkie A. Wilson ${ }^{1,3}$ \\ Departments of ${ }^{1}$ Pharmacology and ${ }^{2}$ Psychiatry, Duke University Medical Center, Durham, North Carolina 27710 \\ and ${ }^{3}$ Neurology Research Laboratory, Veterans Administration Medical Center, Durham, North Carolina 27705
}

Reversal of long-term potentiation by low-frequency stimulation is often referred to as depotentiation. However, it is not clear whether depotentiation induced by low-frequency stimulation and long-term depression (LTD) induced by similar stimuli are distinct phenomena. We have performed a series of experiments in area CA1 of rat hippocampal slices in which a single pattern of theta-burst (TB) stimulation (Larson, et al., 1986; Staubli and Lynch, 1987) was found to produce either LTP or reversal of LTP depending on the intensity of the stimulation. TB stimulation (10 trains consisting of 4 pulses at $100 \mathrm{~Hz}, 200 \mathrm{msec}$ apart) delivered at test pulse-intensity induced LTP. However, the same stimulation delivered at a higher intensity (10 times that of the test pulse, evoking a maximal response) did not induce LTP or depression of control responses, but produced lasting depression of previously potentiated responses. This reversal of LTP (TB depotentiation) was observed when the stimulus was delivered between 0.5 and $110 \mathrm{~min}$ after induction of LTP. TB depotentiation was reversible, cumulative and saturable when high-intensity TB trains were delivered in succession. TB depotentiation was also blocked by antagonists at NMDA receptors. Low-frequency $(1 \mathrm{~Hz})$ stimulation induced LTD, indicating that responses were not already maximally depressed. In addition, high-intensity TB stimulation did not reverse LTD. These results suggest that depotentiation induced by maximal TB stimulation and LTD induced by low-frequency stimulation are distinct phenomena, yet share some characteristics common to forms synaptic plasticity mediated by NMDA receptor activation.

[Key words: long-term potentiation, long-term depression, depotentiation, theta-burst, NMDA receptor, hippocampus]

While long-term potentiation (LTP) has been studied as a candidate learning mechanism for some time, it has only recently become apparent that this increase in synaptic strength is reversible. Long-term decreases in synaptic strength (long-term depression; LTD) may provide a mechanism of "forgetting," and prevent saturation of the storage capacity of the network. A great deal has been learned recently about the mechanisms in-

Received Oct. 11, 1994; revised Feb. 10, 1995; accepted Mar. 10, 1995.

This work was supported by Grant NS-17771 and the VA Medical Center, Durham, North Carolina.

Correspondence should be addressed to Wilkie A. Wilson, Ph.D., Veterans Administration Medical Center, 508 Fulton Street, Building 16, Room 25, Durham, NC 27705.

Copyright (C) 1995 Society for Neuroscience $0270-6474 / 95 / 155402-09 \$ 05.00 / 0$ volved in the induction and expression of LTD (Dudek and Bear, 1992; Mulkey and Malenka, 1992; Malenka and Nicoll, 1993; Mulkey et al., 1993; Bear and Malenka, 1994; Linden, 1994). Interestingly, many of the same requirements for induction of LTP (e.g., NMDA receptor activation and calcium influx) are also required for induction of LTD (reviewed in Bear and Malenka, 1994; Linden, 1994). This implies that subtle differences in activation conditions can result in very different changes in synaptic strength.

"Depotentiation" refers to the reversal of synaptic strength from the potentiated state to pre-LTP levels. Depotentiation has received considerably less attention than LTD, perhaps because depotentiation is often induced with the same low-frequency stimuli that can also depress naive synapses. Therefore, it has not becn clear that depotentiation and LTD are distinct phenomena. Low-frequency stimulation may depress potentiated responses, while producing little (Barrioneuvo et al., 1980; Fujii et al., 1991) or no (Staubli and Lynch, 1990; Larson et al., 1993; Bortolotto et al., 1994; O'Dell and Kandel, 1994) depression of control responses. However, since similar low-frequency stimulation can depress control responses under some circumstances, the reason LTD was not observed in these studies is unclear (Bear and Malenka, 1994). For example, recent studies suggest that the cxpression of LTD versus depotentiation is dependent on the age of the animal; LTD and depotentiation were observed in young animals, but only depotentiation was observed in adult animals (Dudek and Bear, 1993; O'Dell and Kandel, 1994). This distinction would be made clearer if it could be shown that a stimulus protocol induces depression of potentiated responses but not control responses, in a preparation where depression of control responses (by low-frequency stimulation) is robust.

We have studied the effects of low-and high-intensity thetaburst (TB) stimulation in area CA1 of rat hippocampal slices. In light of previous reports (Larson and Lynch, 1986; Larson et al., 1986; Diamond et al., 1988) we expected that TB stimulation above a certain intensity would always induce LTP. However, while low-intensity TB stimulation did induce saturable LTP, high-intensity TB stimulation unexpectedly produced no lasting change of control responses. Instead, high-intensity TB stimulation induced lasting depotentiation of previously potentiated responses. In contrast, low-frequency stimulation produced LTD of control responses, dcmonstrating that control responses were not fully depressed. This observation distinguishes depotentiation from ITD in a preparation where LTD is robust (Barrionuevo, et al., 1980; Staubli and Lynch, 1990; Fujii et al., 1991; Dudek and Bear, 1993; O'Dell and Kandel, 1994). Like both LTP and LTD (reviewed in Malenka and Nicoll, 1993), TB de- 


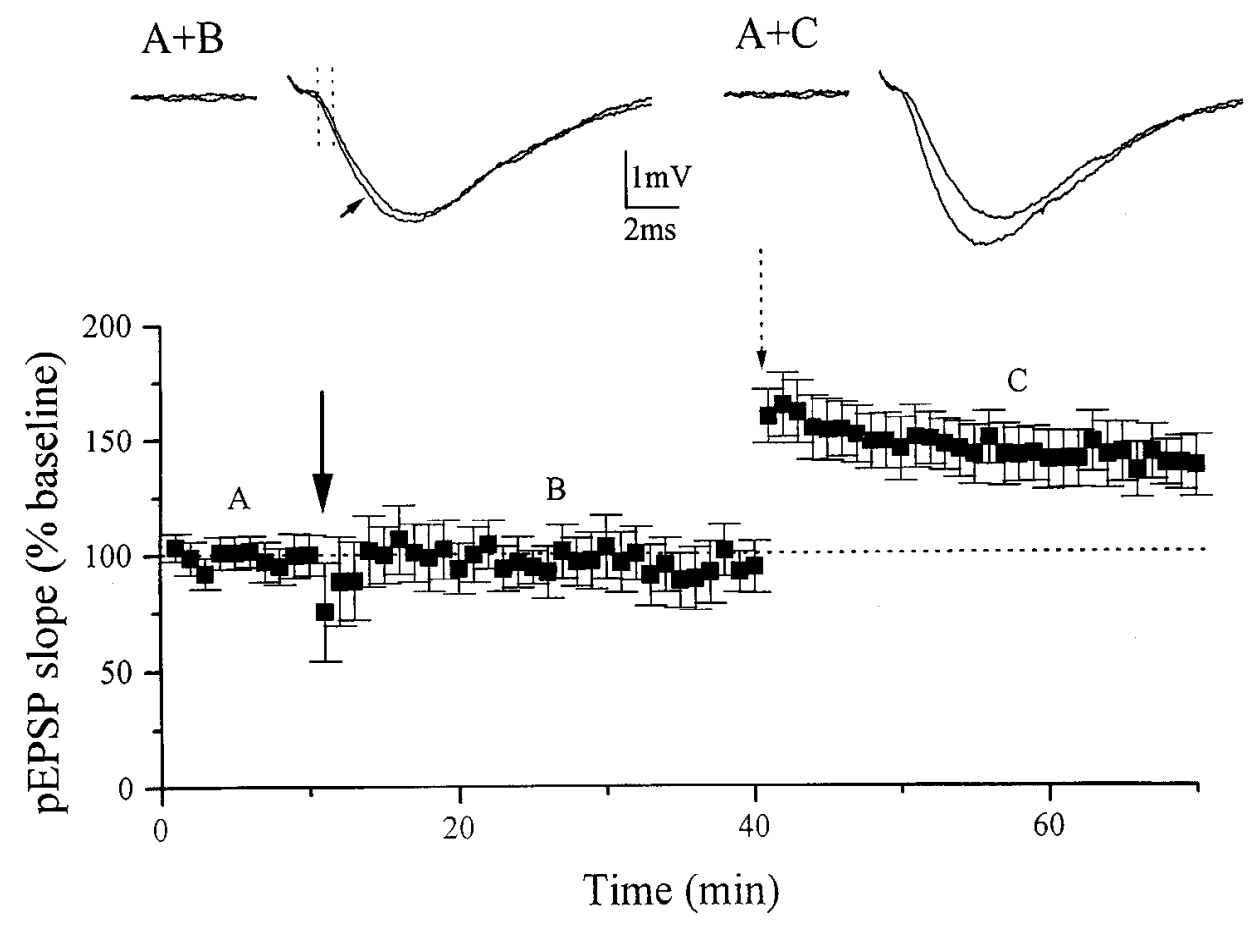

Figure 1. High-intensity theta-burst (TB) stimulation does not induce longterm potentiation (LTP). Each point represents the mean \pm SEM of respective time points from identical experiments. Responses were recorded once per minute for $10 \mathrm{~min}$, at which time a high-intensity TB train (heavy arrow) was delivered. High-intensity TB stimulation did not induce potentiation or depression of responses measured at 40 $\min (95 \pm 11 \%$ baseline, $n=8 ; p=$ 0.24 , paired $t$ test). In addition, highintensity $\mathrm{TB}$ stimulation did not interfere with subsequent potentiation by a low-intensity (dashed arrow) TB train (response measured at $70 \mathrm{~min}, 138 \pm$ $13 \%$ baseline, $n=8 ; p<0.01$, paired $t$ test). Waveforms (above) represent an average of five successive sweeps recorded at the time points designated $A-$ $C$. The slope of the pEPSP was measured over a fixed $600 \mu \mathrm{sec}$ time window during the near-linear rising phase of the response (delineated by the parallel dashed lines). Waveforms $A+B$ illustrate the overlay of responses before and after high-intensity TB stimulation (baseline recording denoted by small arrow at the base of the waveform). Waveforms $A+C$ illustrate the potentiated response superimposed upon the baseline response. potentiation was saturable, and was prevented by blockade of NMDA receptors. Incremental increases in stimulus intensity resulted in graded decreases in potentiation and parallel graded increases in depotentiation. Thus, similar patterns of stimulation can induce or reverse LTP when low- or high-intensity stimuli are delivered, respectively.

\section{Materials and Methods}

Slice preparation. Hippocampal slices $(625 \mu \mathrm{m})$ were prepared from 15-22 d old male Sprague-Dawley rats. Rats were anesthetized with halothane, decapitated, and hippocampi were dissected free. Transverse slices were cut on a Mcllwain tissue chopper in a temporal to septal direction, and incubated for at least an hour in artificial cerebral spinal fluid ( $\Lambda \mathrm{CSF}$ ) warmed to $26^{\circ} \mathrm{C}$ and bubbled with $95 \% \mathrm{O}_{2}, 5 \% \mathrm{CO}_{2}(\mathrm{pH}$ 7.4). Slices were then transferred to a submerged recording chamber perfused at $2 \mathrm{ml} / \mathrm{min}$ with warmed $\left(30-32^{\circ} \mathrm{C}\right)$ oxygenated ACSF of the same compusition (mM): $120 \mathrm{NaCl}, 3.3 \mathrm{KCl}, 1.23 \mathrm{NaH}_{2} \mathrm{PO}_{4}, 25 \mathrm{Na}-$ $\mathrm{HCO}_{3}, 0.9 \mathrm{MgSO}_{4}, 2.0 \mathrm{CaCl}_{2}$, and 10 dextrose. The drugs $\mathrm{D}(-)-2-$ amino-5-phosphonovalerate (D-APV) and 7-chlorokynurenic acid (7CK) (Research Biochemicals International, Natick, MA) were prepared in stock solutions (10-100 mM), and then dissolved in ACSF. We combined the noncompetitive antagonist 7-CK (Kemp et al., 1988) with the competitive antagonist D-APV (Collingridge, et al., 1988) to ensure effective blockade of the NMDA receptor during high-intensity TB stimulation.

Stimulation and recording protocols. A monopolar tungsten stimulating electrode was placed in stratum radiatum to activate the Schaffer collateral pathway projecting to $\mathrm{CA} 1$; in some experiments a second stimulating electrode was placed in stratum radiatum to activate a parallel pathway. Glass microelectrodes filled with $150 \mathrm{~mm} \mathrm{NaCl}(2-10$ $\mathrm{M} \Omega$ ) were positioned in stratum radiatum to record field potential responses. Test pulse stimuli (between 50 and $90 \mu \mathrm{A} ; 0.1 \mathrm{msec}$ square pulses) were delivered to evoke a population excitatory postsynaptic potential (pEPSP) slope of approximately $30 \mathrm{~V} / \mathrm{sec}$. On average, this value was $30 \%$ of the maximal response (data not shown); each slice was tested to insure that test responses were not maximal. Maximal responses were evoked between 500 and $900 \mu \mathrm{A}(10$ times the test pulse-intensity). Responses were evoked once per minute at test pulse- intensity until a stable baseline was maintained (usually $15 \mathrm{~min}$ ). LTP was elicited by delivering 10 minitrains (four pulses at $100 \mathrm{~Hz}$ ) 200 msec apart, at test pulse-intensity. Responses were recorded for at least 60 min afterwards. Signals were amplified using a WPI model 750 dual probe amplifier, and responses were displayed and stored on magnetic disk using a Nicolet 310 digital oscilloscope (digitization rate $50 \mathrm{kHz}$ ). Post-train epileptiform activity was monitored using a chart recorder. The slope of the pEPSP was measured using the oscilloscope cursors (no fitting routine was employed) over a fixed $600 \mu \mathrm{sec}$ time window during the near-linear rising phase of the response (Fig. 1).

For depotentiation experiments, a maximal-intensity $(10 \times)$ TB stimulation was delivered at various time points after the induction of LTP. Percentage depotentiation was measured as [ $\Delta$ pEPSP slope of potentiated response $-\Delta$ in pEPSP slope poststimulation $/ \Delta$ pEPSP slope of potentiated response] $\times 100$. In experiments testing the effect of highintensity TB on LTD, LTD was induced in CAl by delivering two series of 600 pulses at $1 \mathrm{~Hz}$ (test pulse-intensity), separated by a $10 \mathrm{~min}$ interval. A high-intensity TB stimulus was then given to the same pathway 10 min later. LTD was measured as percentage change over baseline in pEPSP slope at $1 \mathrm{hr}$. All values are expressed as mean \pm SEM. Statistical comparisons were made using paired or independent Student's $t$ test or one-way ANOVA where appropriate.

\section{Results}

\section{High-intensity $T B$ stimulation does not induce LTP}

In the first set of experiments we confirmed that theta-burst stirnulation at test intensity (which evoked a response about $30 \%$ of the maximum response) reliably induced robust long-term potentiation, similar to that reported previously by others (Larson and Lynch, 1986; Larson et al., 1986; Diamond et al., 1988). Unexpectedly, however, we found that increasing the stimulus intensity to 10 times test intensity (which evoked a maximal response) did not induce LTP. This finding is illustrated in Figure 1, where high- and low-intensity TB stimulation are delivered sequentially to the same pathway. High-intensity TB stimulation induced no lasting change in synaptic transmission (pEPSP slope 


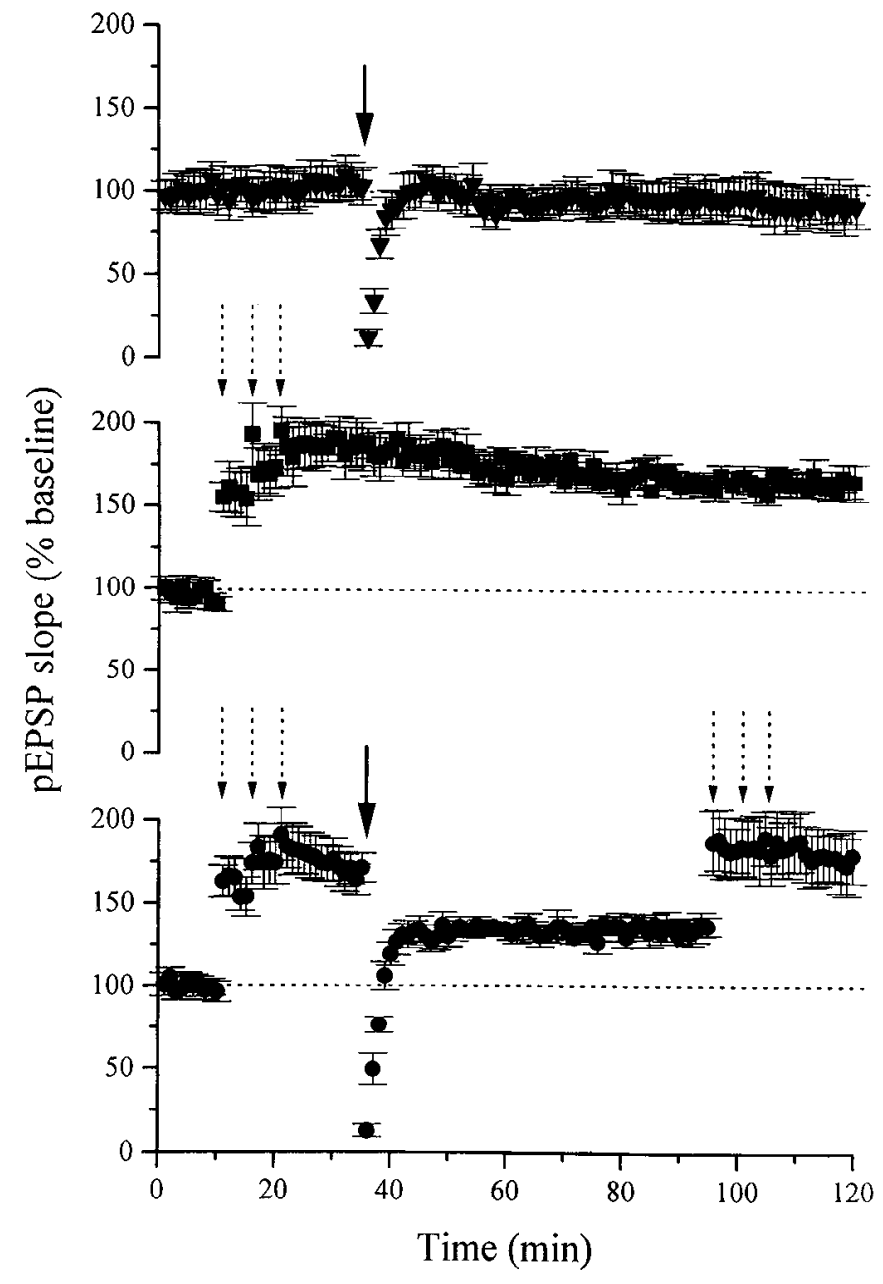

Figure 2. High-intensity TB stimulation depresses potentiated (but not control) responses in a reversible manner. Top (triangles), Time course illustrating that high-intensity TB stimulation does not depress control responses. Responses were recorded once per minute for $35 \mathrm{~min}$, at which time a high-intensity TB train (heavy arrow) was delivered to the same pathway; responses were not significantly different from baseline 80 min later $(92 \pm 11 \%$ of baseline, $n=6 ; \mathrm{p}=0.6$, paired $t$ test $)$ Middle (squares), Time course demonstrating that saturated LTP remains stable. Three low-intensity TB trains delivered in control slices induced LTP that decayed slightly for approximately $30 \mathrm{~min}$ and was stable thereafter $(165 \pm 11 \%$ of baseline after $120 \mathrm{~min} ; n=5 ; p<$ 0.001 , paired $t$ test). In these experiments, one high-intensity TB train delivered $110 \mathrm{~min}$ after LTP induction depressed the potentiated response to $132 \pm 9 \%$ of baseline $(n=5 ; p<0.05$ compared to potentiated responses, paired $t$ test; data not shown). Bottom (circles), TB depotentiation is reversible. LTP was saturated by delivering three lowintensity TB trains (first set dashed arrows; $170 \pm 7 \%$ of control, $n=$ 5); 15 min after saturation, a high-intensity TB train was given to the potentiated pathway (heavy arrow), and LTP was reversed by $54 \pm 9 \%$ $(n=5)$. Responses could be repotentiated with subsequent low-intensity TB trains (second set dashed arrows; resaturated LTP, $179 \pm 16 \%$ of control; $n=5 ; p=0.56$ compared to initial LTP, paired $t$ test). The ability to resaturate LTP at the previous level rules out the possibility that depotentiation is the result of nonspecific tissue damage, and shows that depotentiation is reversible. Each point represents the mean \pm SEM of respective time points from identical experiments.

$95 \pm 11 \%$ of baseline; $n=8, p=0.24$ after $30 \mathrm{~min}$ ). LTP was induced in the same pathway, however, by TB stimulation delivered at test intensity $(138 \pm 13 \%$ of baseline; $n=8, p<$ 0.01 after $30 \mathrm{~min}$ ). These results suggest that high-intensity stimulation fails to induce LTP, but does not prevent later induction of LTP in the same pathway.
In a second set of experiments we tested the effect of lowand high-intensity TB stimulation on two independent pathways. Two stimulating electrodes were placed in the Schaffer collateral/commissural pathway; independent pathways were confirmed by the absence of paired-pulse facilitation between electrodes at test pulse-intensity. First, low-intensity TB stimulation was delivered to one pathway, and this resulted in an increase in pEPSP slope of $158 \pm 9 \%$ of baseline values $(n=13 ; p<$ 0.0001 ). TB stimulation was then given 30 min later to the adjacent pathway at 10 times the test-pulse intensity. This did not induce LTP in this pathway (106 $\pm 6 \%$ of baseline, $n=13 ; p$ $=0.31$ ). High-intensity TB stimulation did not significantly alter the potentiated response in the other pathway $(p=0.54)$, (data not shown).

In a separate group of experiments the order of high- and lowintensity TB stimulation were reversed, to determine whether high-intensity TB in one pathway prevented subsequent induction of LTP in the other. As in the previous experiments, LTP was not induced in the pathway receiving a high-intensity TB stimulus $(105 \pm 8 \%$ of baseline, $n=10 ; p=0.39)$. However, responses recorded from the adjacent pathway were potentiated secondarily by the high-intensity TB stimulus (157 $\pm 9 \%$ baseline, $n=10 ; p<0.05$ ). Because LTP is pathway specific (reviewed in Bliss and Collingridge, 1993) we suspected that this apparent "heterosynaptic" potentiation resulted from stimulus spread occurring with the high-intensity stimulus. This suspicion was contirmed by the observation that paired-pulse facilitation occurred between the two pathways when the stimulus intensity was increased to 10 times the test intensity.

\section{High-intensity $T B$ stimulation reverses $L T P$}

The results of this first set of experiments suggested that increasing the intensity of theta-pattern stimulation prevents induction of LTP. We next tested whether high-intensity TB stimulation disrupts the maintenance of preestablished LTP. In these experiments low-intensity TB stimulation, which induced LTP, was followed at various intervals by high-intensity TB stimulation. Potentiated responses were persistently depotentiated when high-intensity stimulation was delivered between $30 \mathrm{sec}$ and 30 min following the induction of LTP. After $60 \mathrm{~min}$, slices that did not receive high-intensity TB stimulation were potentiated 146 $\pm 6 \%$ of baseline $(n=10)$. Separate groups of slices that did receive high-intensity TB stimulation at time points between 30 $\mathrm{sec}$ and $30 \mathrm{~min}$ were not significantly potentiated at $60 \mathrm{~min}$ compared to the control group [timc, mcan \pm SEM\% of baseline $(n): 30 \mathrm{sec}, 111 \pm 6 \%(6) ; 1 \mathrm{~min}, 123 \pm 8 \%(8) ; 3 \mathrm{~min}$, $110 \pm 3 \%(5) ; 5 \mathrm{~min}, 112 \pm 7 \%(6) ; 10 \mathrm{~min}, 111 \pm 10 \%(4)$; $15 \mathrm{~min}, 94 \pm 9 \%(5)$; $30 \mathrm{~min}, 99 \pm 4 \%$ (6); one-way ANOVA, df $=8, F=5.3, p<0.001$; all pairwise multiple comparisons between control LTP and LTP followed by high-intensity TB stimulation at designated time points, $p<0.05$, Student NewInan Keuls method]. Taken together, these results indicate that high-intensity TB stimulation does not prevent subsequent induction of LTP, but does inhibit maintenance of preestablished LTP.

Depression of potentiated responses could result from a pathological consequence of the high-intensity stimulation; the process of long-term depression (LTD) superimposed on LTP; or from a separate process that reverses the expression of LTP (depotentiation). In order to rule out the first of these possibilities, we first saturated LTP by delivering three low-intensity TB trains (Fig. 2, bottom). [The response $10 \mathrm{~min}$ after the third TB train 
was not significantly potentiated compared to that following the second $\left(\mathrm{LTP}_{1}, 152 \pm 9 \% ; \mathrm{LTP}_{2}, 173 \pm 10 \% ; \mathrm{LTP}_{3}, 170 \pm 7 \%\right.$, $n=6 ; \mathrm{LTP}_{1}$ compared to $\mathrm{LTP}_{2}, p<0.05 ; \mathrm{LTP}_{2}$ compared to $\mathrm{LTP}_{3}, p=0.5$ ), confirming that LTP was saturated]. Following saturation of LTP, a single high-intensity TB train was delivered to produce depression. If depression resulted from an actual reversal of LTP, we expected that we would be able to resaturate LTP with additional low-intensity TB trains (Mulkey and Malenka, 1993). This was indeed the case, as additional low-intensity trains returned responses to their previously saturated levels (Fig. 2, bottom; resaturated LTP, $179 \pm 16 \%$ of baseline; $n=$ $6 ; p=0.56)$. In fact, LTP apparently resaturated following the first train (Fig. 2, bottom). Three low-intensity TB trains delivered in control slices induced LTP that decayed slightly for approximately $30 \mathrm{~min}$ and was stable thereafter $(165 \pm 11 \%$ of baseline after $120 \mathrm{~min} ; n=5 ; p<0.001$; Fig. 2, middle). In these experiments, one high-intensity TB train delivered even after $100 \mathrm{~min}$ depressed the potentiated response to $132 \pm 9 \%$ of baseline ( $n=5 ; p<0.05$ compared to potentiated responses; data not shown). These results demonstrate that like LTP and LTD, TB depotentiation can be reversed.

As was shown previously (Fig. 1), control responses were not depressed by high-intensity TB stimulation (Fig. 2, top; response 80 min post-high-intensity $\mathrm{TB}, 92 \pm 11 \%$ of baseline, $n=6$; $p=0.6$ ). This suggested that reversal of LTP by high-intensity TB stimulation was distinct from LTD. However, in order to definitively distinguish depotentiation from LTD, it was necessary to demonstrate that control responses had the potential to become depressed in these slices; that is, that they could express LTD. In addition, it seemed reasonable to test the possibility that high-intensity TB stimulation could also reverse LTD. Both of these questions were addressed in a set of experiments where low frequency, low-intensity stimulation (LFS, $1 \mathrm{~Hz}, 600$ pulses) was followed by high-intensity TB stimulation. LFS induced significant, persistent LTD, as previously reported (Fig. 3, top; response $80 \mathrm{~min}$ following low-frequency stimulation, $45 \pm 15 \%$ baseline, $n=5$; $p<0.05$ ) (Dunwiddie and Lynch, 1978; Dudek and Bear, 1992). High intensity TB stimulation following LTD induction did not reverse low-frequency-induced depression (Fig. 3, bottom; response $70 \mathrm{~min}$ following high-intensity TB, $32 \pm 12 \%$ of baseline, $n=6 ; p<0.001)$. These results demonstrated that control responses could be depressed by low-frequency stimulation, but not by high-intensity TB stimulation. In addition, they show that high-intensity TB stimulation reverses LTP but not LTD. This last result supports the results of previous studies which indicate that the mechanisms involved in the maintenance of LTP and LTD are different, despite the similar requirements for induction (Malinow et al., 1988; Mulkey et al., 1993).

The previous experiments indicated that depotentiation induced by high-intensity TB stimulation is a form of synaptic plasticity which is different from LTP and LTD. We performed additional experiments to determine if this form of plasticity shares any characteristics of LTP and LTD. Both LTP and LTD can be induced cumulatively; that is, multiple high- or low-frequency trains produce cumulative potentiation or depression until a saturation point is reached (Mulkey and Malenka, 1992). We therefore tested the effect of multiple high-intensity TB trains on potentiated responses. As shown in Figure 4 (top), multiple high-intensity TB trains induced cumulative depotentiation; after three such trains the response was depressed to the prepotentiated (control) level (response $20 \mathrm{~min}$ after third de-

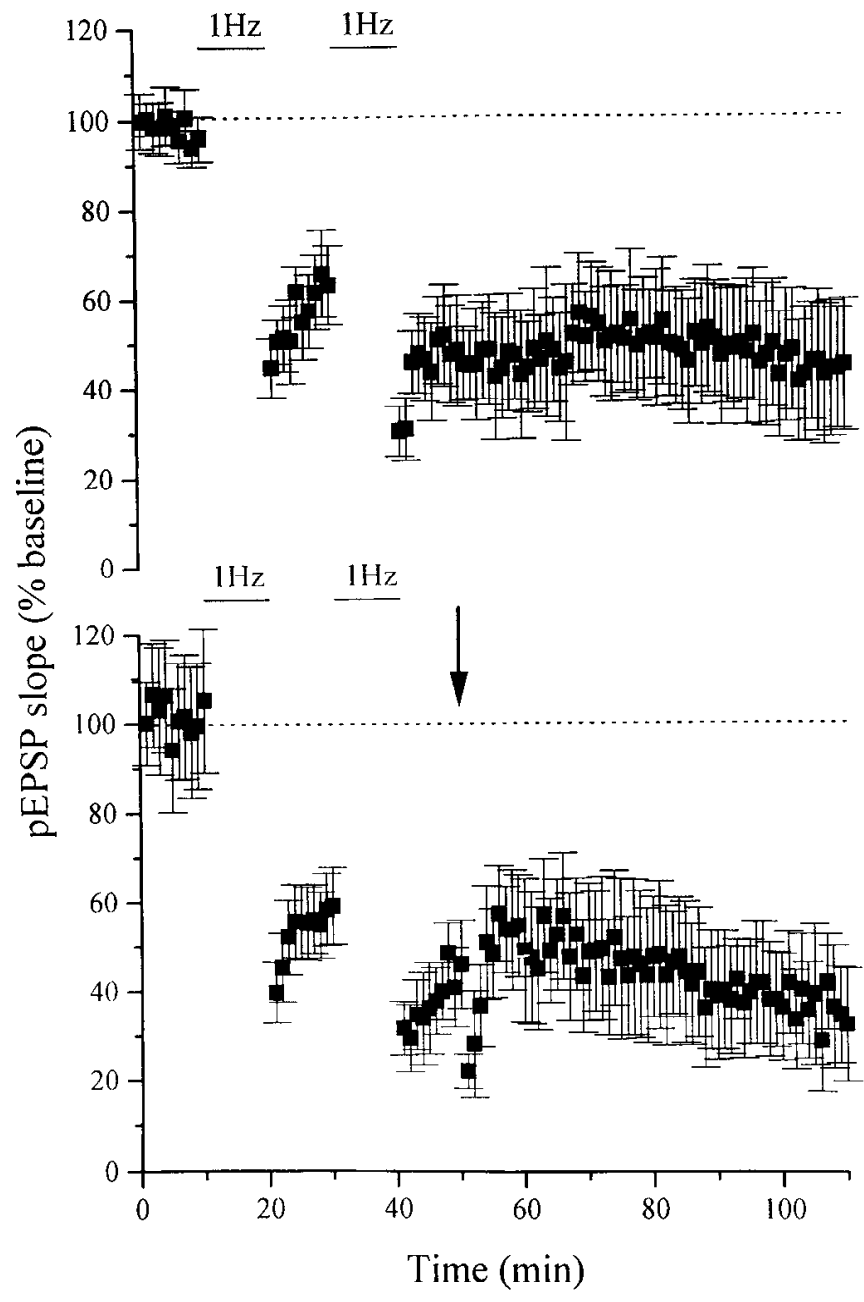

Figure 3. High-intensity TB stimulation does not reverse long-term depression (LTD). Top, LTD was induced by delivering two low-frequency stimulus (LFS) trains ( $1 \mathrm{~Hz}$ for $10 \mathrm{~min}$, designated by bars), and responses remained depressed for $80 \min (45 \pm 15 \%$ baseline, $n$ $-5 ; p<0.05$, paired $t$ test). Bottom, In a separate group of experiments, a high-intensity TB train delivered 10 min after the second LFS train (heavy arrow) did not reverse LTD $(33 \pm 13 \%$ of baseline, $n=$ $6 ; p<0.001$, paired $t$ test); LTD followed by a high-intensity TB was not significantly different from control LTD $(p=0.45$, independent $t$ test). Each point represents the mean \pm SEM of respective time points from identical experiments.

potentiation stimulus, $108 \pm 14 \%$ of baseline, $n=5 ; p=0.3$ ). It was evident that the first high-intensity TB train induced more depotentiation than the subsequent trains, such that the third train induced very little additional depotentiation. These results indicate that, like LTP and LTD, depotentiation induced by highintensity $\mathrm{TB}$ stimulation is cumulative and saturable, with the level of transmission at saturation being the prepotentiated level.

Both LTP and LTD require activation of NMDA receptors for induction (Collingridge et al., 1983; Stringer and Guyenet, 1983; Coan et al., 1987; Dudek and Bear, 1992; Mulkey and Malenka, 1992). We therefore tested the possibility that depotentiation also required NMDA receptor activation. In these experiments the NMDA receptor antagonists D-APV and 7-CK were present when multiple high-intensity TB trains were delivered. Addition of D-APV and 7-CK alone slightly depressed pEPSPs, suggesting that NMDA receptors contributed to test responses (Fig. 4, bottom). As is shown in Figure 4, depotentiation was not in- 


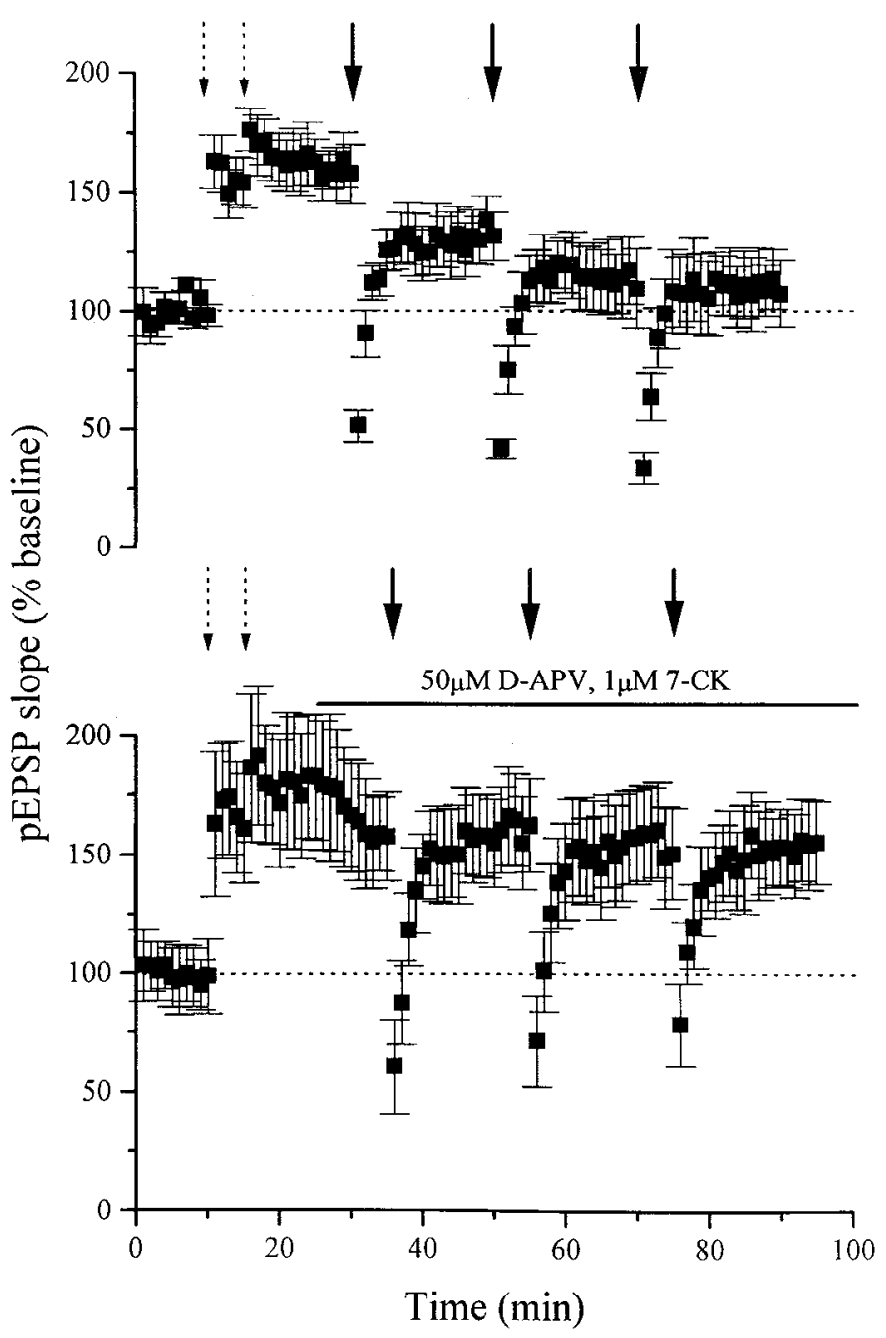

Figure 4. TB depotentiation is cumulative, saturable, and is blocked by NMDA receptor antagonists. Top, LTP was induced (single dashed arrows), at which time three successive high-intensity TB trains were delivered at 20 min intervals (heavy arrows); depotentiation accumulated with each stimulation, and approached saturation near the prepotentiated level (108 $\pm 14 \%$ of baseline, $n=5 ; p=0.3$, paired $t$ test $)$ Bottom, Multiple trains given in the presence of D-APV and 7-CK (bar indicates duration of bath application) did not induce depotentiation (response at $35 \mathrm{~min}, 153 \pm 18 \%$ of baseline; at $100 \mathrm{~min}, 150 \pm 8 \%$ of baseline, $n=5 ; p=0.7$, paired $t$ test). Control responses were slightly depressed with application of NMDA receptor antagonists. Each point represents the mean \pm SEM of respective time points from identical experiments.

duced by high-intensity TB trains in the presence of D-APV and 7 -CK (15 min after saturation of LTP, $153 \pm 18 \%$ of baseline, $n=5, p<0.001 ; 20$ min after third high-intensity TB train in the presence of NMDA antagonists, $150 \pm 8 \%$ of baseline, $n$ $=5 ; p=0.7$ ). Thus TB depotentiation, like LTP and LTD, requires activation of NMDA receptors.

The previous experiments indicated that low-intensity TB stimulation induced LTP, whereas high-intensity TB stimulation reversed LTP. We therefore tested a range of intensities for both induction of LTP and depotentiation by TB stimulation, to determine whether induction of these forms of TB-induced plasticity was graded with respect to stimulus intensity. As shown in Figure 5 (open triangles), the magnitude of depotentiation (expressed as percentage depotentiation; see Materials and
Methods) by TB stimulation increased with increasing stimulus intensity. Although TB stimulation at five times the test-intensity induced depression of potentiated responses, this decrease was not significantly greater than the normal decrement of responses following saturation of LTP (Fig. 5, open triangles; percentage change in potentiation following $1 \times \mathrm{TB}$ stimulation, $3 \pm 15 \%$, $n=11$; following $5 \times$ TB stimulation: $39 \pm 14 \%, n=6 ; p=$ $0.14)$. Significant depotentiation occurred at $10 \times$ intensity (54 $\pm 9 \%, n=5 ; p=0.046$ ), while TB depotentiation at 15 times the test-intensity induced even greater depotentiation, completely returning responses to the control level (percentage depotentiation, $15 \times, 107 \pm 11 \%, n=6 ; p<0.001)$.

As shown in Figure 5 (solid squares), the magnitude of I.TP was graded downward with respect to stimulus intensity. TB stimulation at test intensity $(1 \times)$ resulted in potentiation to 167 $\pm 17 \%$ of the baseline. TB stimulation at 5 times intensity induced less LTP than $1 \times(130 \pm 11 \%$ of baseline, $n=5, p=$ 0.10 ), while 10 times intensity failed to induce LTP (105 \pm $14 \%$ of baseline, $n=10 ; p<0.05$ compared to $1 \times)$. These results suggest that both induction of LTP and depotentiation by TB stimulation are graded with respect to stimulus intensity. Furthermore, the stimulus threshold for either the failure to induce LTP, or the reversal of LTP, converges at 10 times the test pulse-intensity.

Afterdischarges often accompanied high-intensity TB stimulation during depotentiation experiments. In the final set of experiments we differentiated between the effects of the stimulation and afterdischarge by elevating the extracellular $\mathrm{Ca}^{2+}$ concentration to eliminate the afterdischarge. When the $\mathrm{Ca}^{2+}$ concentration was raised to $4.0 \mathrm{~mm}$ during the high-intensity TB train, depotentiation occurred in the absence of afterdischarges (Fig. 6). Under these conditions depotentiation was in fact significantly greater than that in standard $\left(2.0 \mathrm{mM} \mathrm{Ca}^{2+}\right) \mathrm{ACSF}$ (percentage depotentiation: $2.0 \mathrm{mM} \mathrm{Ca}^{2}, 54 \pm 9 \%, n=5 ; 4$ $\left.\mathrm{mM} \mathrm{Ca}{ }^{2+}, 81 \pm 5 \%, n=6 ; p<0.05\right)$. These results indicate that afterdischarges are not required for depotentiation. In addition, the finding that depotentiation was significantly greater in higher $\mathrm{Ca}^{2+}$ is generally consistent with other studies linking NMDA receptor-mediated postsynaptic calcium entry to induction of both LTP (Dunwiddie and Lynch, 1979; Huang et al., 1988; Mulkeen et al., 1988) and LID (Mulkey and Malenka, 1992).

\section{Discussion}

The present study clarifies the distinction between depotentiation and the induction of LTD at previously potentiated synapses, which has also been called depotentiation. This is appropriate, since it is clear that the (usually low-frequency) stimuli used to induce LTD actually reverse the process of LTP (Mulkey and Malenka, 1993; Barrionuevo et al., 1980; Staubli and Lynch, 1990; Fujii et al., 1991; Bashir et al., 1993; Larson et al., 1993; Bortolotto et al., 1994). Some studies have demonstrated that this type of stimulus produces little (Fujii et al., 1991) or no (Staubli and Lynch, 1990; Larson et al., 1993; Bortolotto et al., 1994) depression of control responses. Although unlikely, it was possible that control responses were maximally depressed under those particular experimental conditions. A more likely possibility is that the induction of LTD at naive synapses by this type of stimulation is age-dependent, such that low-frequency-induced depression is more likely to occur in younger animals (Dudek and Bear, 1993; O'Dell and Kandel, 1994). In addition, the threshold for low-frequency-induced depression may be low- 


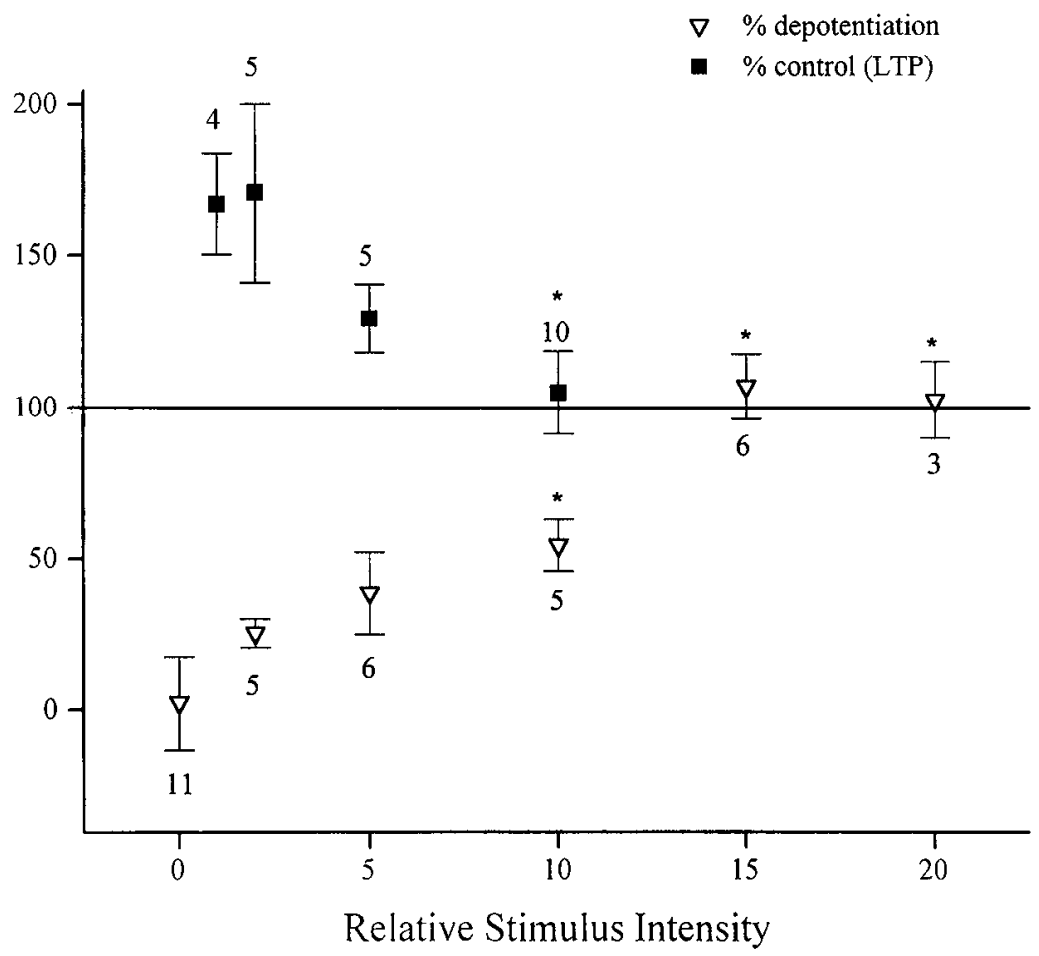

Figure 5. Both TB potentiation and depotentiation are graded with respect to stimulus intensity. TB potentiation (solid squares) decreases with increasing stimulus intensity, such that the threshold for the failure to induce LTP occurred at $10 \times$ intensity (LTP by $1 \times$ TB stimulus $(\%$ of baseline), $167 \pm 17 \% ; 2 \times, 171 \pm 30 \%, p=0.92 ; 5 \times, 130 \pm 11 \%, p=0.10 ; 10 \times, 105 \pm 14 \% ; p<0.05 ; p$ values are the results of independent $t$ tests comparing LTP to control LTP; the asterisk indicates significance compared to control LTP). TB depotentiation (open triangles), on the other hand, increases with increasing stimulus intensity. Relative stimulus intensity " 0 " represents the decrement in potentiation 45 min following saturated LTP without additional stimulation; all other points represent percentage depotentiation at the respective stimulus intensities (percentage depotentiation was measured as the average of experimental time points 45 min following the depotentiating stimulus). In these experiments, saturated LTP was followed 15 min later by a TB train at stimulus intensities ranging from 2 to 20 times test intensity. Increasing stimulus intensity resulted in increased depotentiation, with 10 times test intensity the threshold for significant depotentiation [control $(0), 3 \pm 15 \% ; 2 \times, 25 \pm 5 \%, p=0.34 ;$ $5 \times, 39 \pm 14 \%, p=0.14 ; 10 \times, 54 \pm 9 \%, p<0.05, p$ values are results of independent $t$ tests comparing depotentiation to the control decrement; the asterisk indicates significance compared to control]. Full depotentiation of responses plateaued at $15 \times$ intensity $(15 \times$ TB depotentiating stimulus, $107 \pm 11 \%, p<0.001 ; 20 \times$ TB stimulus, $103 \pm 13 \%, p<0.01$, independent $t$ test). These results indicate that 10 times test-pulse intensity is the stimulus threshold for either the failure to induce LTP, or reversal of LTP by TB stimulation; $n$ values appear on the graph for each data point.
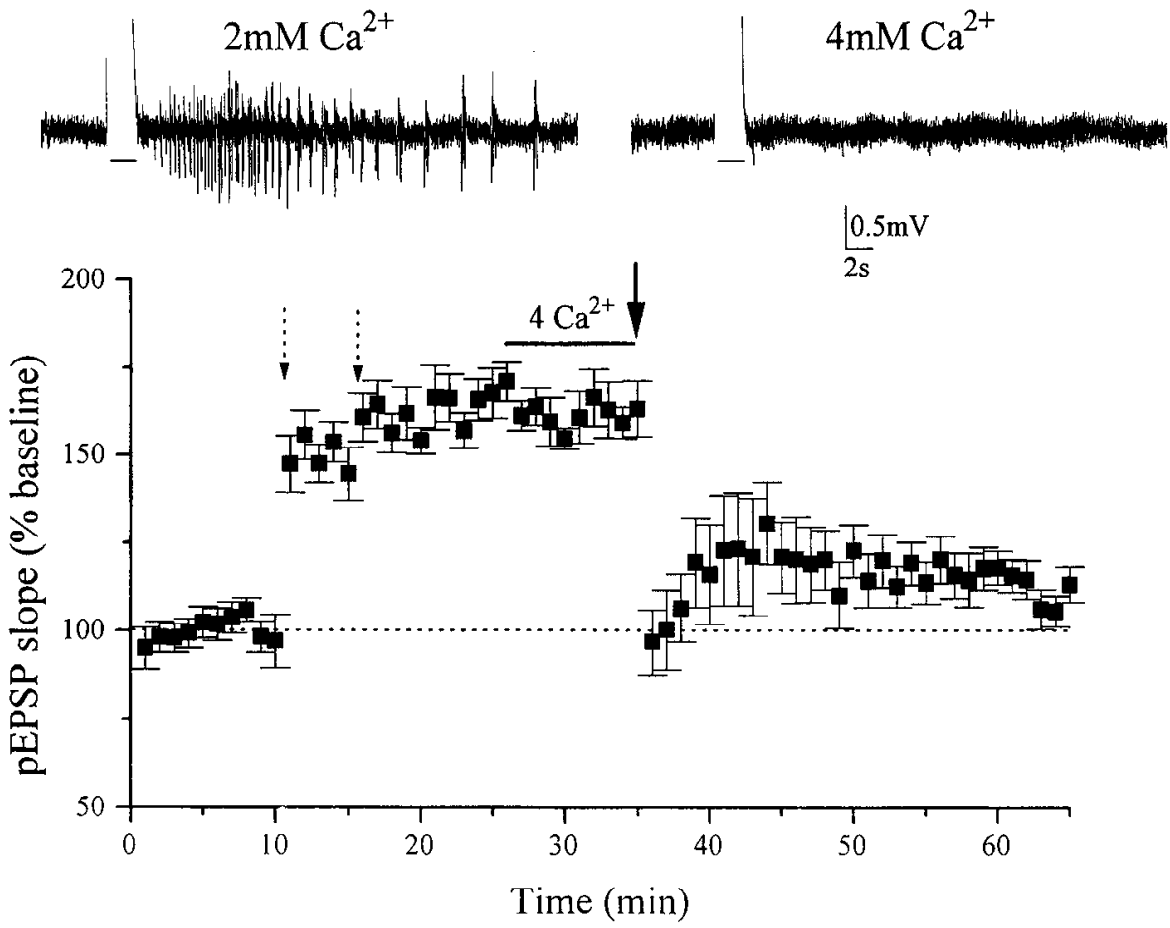

Figure 6 Afterdischarges are not required for TB depotentiation. The chart record illustrates sample afterdischarges recorded in CA1 following a highintensity TB train in normal ACSF (top left), and the absence of afterdischarges in $4 \mathrm{mM} \mathrm{Ca}^{2+} \mathrm{ACSF}$ (top right; chart record taken from a single slice). In separate experiments, LTP was induced by low-intensity TB stimulation (dashed arrows; $163 \pm 8 \%$ baseline at $35 \min , n=6$ ). The high-intensity TB train (heavy arrow) was then delivered in the presence of $4 \mathrm{mM} \mathrm{Ca}^{2+} \mathrm{ACSF}$ (bar indicates perfusion time), which eliminated afterdischarges. The response measured at $65 \mathrm{~min}$ was reduced to $113 \pm 5 \%$ baseline $(n=6$; $p<0.0001$ compared to potentiated response, paired $t$ test). This represented $81 \pm 5 \%$ depotentiation $(n=6)$. This result indicates that depotentiation by high-intensity TB stimulation does not require afterdischarges. 
er at potentiated synapses than at naive synapses (Wexler and Stanton, 1993). Therefore, it is possible that LTD and depotentiation are actually the same process, but that in adult preparations which have undergone potentiation, the threshold for lowfrequency-induced depression is lowered.

The results of the present study, however, indicate that there is a second route to LTP reversal which is distinct from lowfrequency-induced depression, and restricted only to potentiated synapses. TB depotentiation differs from low-frequency-induced depression in that high-intensity TB trains reversed LTP with only a 2 sec stimulation; low-frequency-induced depression requires minutes of synaptic activation. More importantly, we have demonstrated three distinct forms of plasticity in the same preparation, one in which low-frequency LTD is readily induced. Surprisingly, depotentiation can be induced by the same stimulus pattern used to induce LTP, but at a higher stimulus intensity. This result is somewhat counterintuitive, since earlier studies characterizing LTP in the hippocampus have demonstrated that very low stimulus intensities (threshold for evoking responses) do not induce LTP, while stimulus intensities severalfold higher do (Yamamoto and Sawada, 1981).

We are aware of few studies in the hippocampus which have reported that stimulation like that used to induce LTP either does not produce LTP, or reverses preestablished LTP. In one study, Harrison and Alger (1992) found that LTP (100 Hz, $2 \mathrm{sec}$ ) was partially depressed when followed by the same stimulation delivered at $4-8 \times$ the intensity. However, depression occurred in only about half of the trials, and, in some cases, neuronal responsiveness was completely abolished. Bashir and Collingridge (1992) demonstrated a transient, NMDA receptor-dependent depression of naive responses following LTP stimulation delivered at double test-intensity $(100 \mathrm{~Hz}, 1 \mathrm{sec})$, in the presence of pic-

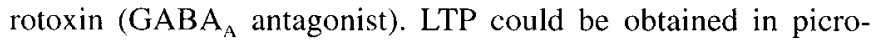
toxin with the same stimulation at test pulse-intensity. Their results differ from ours, as they found that double test-intensity stimulation in the presence of picrotoxin resulted in heterosynaptic depression; in our study, high-intensity TB stimulation did not depress control responses in the same (or separate) pathways. Unfortunately, these authors did not examine the effects of high-intensity stimulation on previously potentiated synapses. These results complement our findings, however, because they also suggest that during relatively high-intensity stimulation or conditions which increase neuronal excitability, induction of LTP is prevented.

O'Dell and Kandel (1994) recently reported that $5 \mathrm{~Hz}$ stimulation reverses LTP in adult guinea pig slices, while the same stimulation actually induced slight potentiation at naive synapses. Like previous studies, however (e.g., Fujii et al., 1991; Bortolotto et al. 1994), they did not observe low-frequency $(1 \mathrm{~Hz})$ LTD in the same slices (LTD was observed in slices from young animals). These authors did not report the effects of $5 \mathrm{~Hz}$ stimulation on naive or potentiated synapses in young rats; however, others have shown that such stimulation depresses naive responses in young animals (Dudek and Bear, 1992; Bolshakov and Siegelbaum, 1994). In any case, the depotentiation observed by O'Dell and Kandel in adult guinea pigs appears to be distinct from that observed in the present study, since depotentiation in their case became ineffective within an hour of LTP induction, and depotentiation in the present study was fully effective for over an hour postinduction (O'Dell and Kandel, 1994).

The results of this and previous studies suggest that activation of NMDA receptors can trigger induction of LTP, LTD, or de- potentiation (reviewed in Malenka and Nicoll, 1993). The dependence of TB depotentiation on NMDA receptor activation demonstrated here implies that calcium influx may also be important for this form of plasticity, although we have not directly tested this. One unresolved question is how can a single trigger mechanism induce three different forms of plasticity (Lisman, 1989; Malenka and Nicoll, 1993; Teyler et al., 1994)? Lisman (1989) has proposed a model where opposite changes in synaptic strength occur following different levels of calcium influx (see also Bienenstock et al., 1982). The results of this study suggest that such models may have to be modified to include the prior state of the synapse as well. The duration of the calcium elevation may also have to be considered, as depotentiation in the present study was induced by relatively brief stimuli. High-intensity stimulation will activate a larger population of synapses than low-intensity stimulation. We speculate that this results in a larger calcium influx during the high-intensity TB train, a proposition which can be experimentally tested. At this point we can only speculate on the molecular mechanism(s) responsible for TB depotentiation. LTD, and the reversal of potentiation by $5 \mathrm{~Hz}$ stimulation in adult animals requires activity of protein phosphatases (Mulkey et al., 1993; O'Dell and Kandel, 1994). The possible role of protein phosphatases in TB depotentiation is worth investigating.

Many manipulations interfere with induction of LTP, but there are relatively few which can selectively erase established LTP. One such manipulation may be inhibition of heme oxygenase (Stevens and Wang, 1993). Inhibition of heme oxygenase also prevents induction of LTP, yet does not alter control responses, and does not affect low-frequency LTD. These results coincide directly with the effects of high-intensity TB stimulation on control responses, LTP, and LTD. Other studies have demonstrated that inhibition of protein kinases suppresses established LTP (Malinow et al., 1988; Matthies et al., 1991) in a reversible manner (Malinow et al., 1988). Finally, seizure activity interferes with maintenance of LTP in vivo (Hesse and Teyler, 1976), although the molecular mechanism of this effect is unknown.

One implication of depotentiation (as opposed to LTD) is that very few synapses in unstimulated slice preparations must be in a potentiated state, otherwise the depotentiating stimulus would depress control responses. It is possible that potentiation can only be reversed at recently potentiated synapses, implying that very few synapses in intact animals (and naive hippocampal slices) are in a recently potentiated, and hence susceptible state. In other words, even if a large percentage of synapses were in a potentiated state, one would expect a small percentage of these to have been potentiated very recently. We were able to reverse LTP up to 110 min after induction, but do not know if depotentiation would occur after several hours. It is also possible that the slicing procedure itself somehow resets synaptic strength at potentiated synapses to a baseline level.

What might be the functional implications of high-intensity, TB depotentiation? The conditions necessary for depotentiation observed in the present study (burst-like activation of a large number of afferent fibers) might occur physiologically with synchronized firing, as seen during seizure activity. Inhibition of potentiation with very strong levels of synaptic activation may serve a protective function to prevent generalized, nonspecific potentiation and subsequent impairment of network functioning following seizures. Assuming that LTP represents the cellular correlate of memory, and that only recently consolidated LTP is disrupted, the consequences of seizure activity would be rela- 
tively benign: recent memory would be lost (erased), but consolidated information would be intact, and new information could be stored right away. In contrast, if seizure activity induced (and possibly saturated) LTP at a large proportion of available unpotentiated synapses, the consequences would be much more severe: stored information would be contaminated by the "noise" of nonspecific LTP, and new information could not be stored until some synapses hecame available for specific potentiation. Memory loss has in fact been documented for epileptic or electroconvulsive therapy-induced seizures (Halgren and Wilson, 1985; Squire, 1986; Thompson, 1991). Moreover, studies have demonstrated that evoking electrographic seizures, or mimicking the extracellular conditions during seizures inhibits the induction (Moore et al., 1993) or maintenance of LTP (Hesse and Teyler, 1976; Harrison and Alger, 1992). Although in this study we show that TB depotentiation occurs in the absence of epileptiform activity, this does not preclude the possibility that repetitive neuronal firing of sufficient magnitude (as in a seizure) could affect plasticity in the intact animal.

In summary, high-intensity TB stimulation exceeded the level of synaptic activation required to enhance synaptic strength, yet did not result in potentiation. In addition, this level of stimulation reversed preestablished LTP in an NMDA receptor-dependent fashion. These findings are generally consistent with previous findings which show that differences in the level and pattern of synaptic activation influence the threshold for induction of plastic changes (Christie and Abraham, 1992; Huang et al., 1992; Wexler and Stanton, 1993), and may determine the form of plasticity expressed.

\section{References}

Barrionuevo G, Schottler F, Lynch G (1980) The effects of repetitive low frequency stimulation on control and "potentiated" synaptic responses in the hippocannpus. Life Sciences 27:2385-2391.

Bashir ZI, Collingridge GL (1992) NMDA receptor-dependent transient homo- and heterosynaptic depression in picrotoxin-treated hippocampal slices. Eur J Neurosci 4:485-490.

Bashir ZI, Jane DE, Sunter DC, Watkins JC, Collingridge GL (1993) Metabotropic glutamate receptors contribute to the induction of longterm depression in the $\mathrm{CA} 1$ region of the hippocampus. Eur $\mathbf{J}$ Pharmacol 239:265-266.

Bear MF, Malenka RC (1994) Synaptic plasticity: LTP and LTD. Curr Opinion Neurobiol 4:389-399.

Bienenstock El, Cooper LN, Munro PW (1982) Theory for the development of ncuron selectivity: orientation specificity and binocular interaction in visual cortex. J Neurosci 2:32-48.

Bliss TVP, Collingridge GL (1993) A synaptic model of memory: longterm potentiation in the hippocampus. Nature 361:31-39.

Bolshakov VY, Siegelbaum SA (1994) Postsynaptic induction and presynaptic expression of long-term depression. Science 264:1148-1 152

Bortolotto ZA, Bashir ZI, Davies CH, Collingridge GL (1994) A molecular switch activated by metabotropic glutamate receptors regulates induction of long-term potentiation. Nature 368:740-743.

Christie BR, Abraham WC (1992) Priming of associative long-term depression in the dentate gyrus by theta frequency synaptic activity. Neuron 9:79-84.

Coan EJ, Saywood W, Collingridge GL (1987) MK-801 blocks NMDA receptor-mediated synaptic transmission and long-term potentiation in rat hippocampal slices. Neurosci Lett 80:111-114.

Collingridge GL, Kehl SJ, McLennan H (1983) Excitatory amino acids in synaptic transmission in the Schaffer collateral-commissural pathway of the rat hippocampus. J Physiol (Lond) 334:33-46.

Collingridge GL, Herron CE, Lester RAJ (1988) Frequency dependent $N$-methyl-D-aspartate receptor mediated synaptic transmission in the rat hippocampus. J Physiol 399:301-312.

Diamond DM, Dunwiddie TV, Rose GM (1988) Characteristics of hippocampal primed burst potentiation in vitro and in the awake rat. $J$ Neurosci 8:4079-4088.

Dudek SM, Bear MF (1992) Homosynaptic long-term depression in area $\mathrm{CA} 1$ of hippocampus and effects of $N$-methyl-D-aspartate receptor blockade. Proc Natl Acad Sci USA 89:4363-4367.

Dudek SM, Bear MF (1993) Bidirectional long-term modification of synaptic effectiveness in the adult and immature hippocampus. J Neurosci 13:2910-2918.

Dunwiddie TV, Lynch G (1978) Long-term potentiation and depression of synaptic responses in the rat hippocampus: localization and frequency dependency. J Physiol (Lond) 276:353-367.

Dunwiddie TV, Lynch G (1979) The relationship between extracellular calcium concentrations and hippocampal long-term potentiation. Brain Res 169:103-110.

Fujii S, Saito K, Miyakawa H, Ito K, Kato, H (1991) Reversal of longterm potentiation-(depotentiation) induced by tetanus stimulation of the input to CAl neurons of guinea pig hippocampal slices. Brain Res 555:112-122.

Halgren E, Wilson CL (1985) Recall deficits produced by afterdischarges in the human hippocampal formation and amygdala. Electroencephalogr Clin Neurophysiol 61:375-380.

Harrison CM, Alger BE (1992) Perfusion with high potassium plus glutamate can cause LTP erasure or persistent loss of neuronal responsiveness in the CA1 region of the hippocampal slice. Brain Res 602:175-179.

Hesse GW, Teyler TJ (1976) Reversible loss of hippocampal long-term potentiation following electroconvulsive seizures. Nature 264:562564.

Huang Y, Gustafsson B, Wigstrom H (1988) Facilitation of hippocampal long-term potentiation in slices perfused with high concentrations of calcium. Brain Res 456:88-94.

Huang Y, Colino A, Selig DK, Malenka RC (1992) The influence of prior synaptic activity on the induction of long-term potentiation. Science 255:730-733.

Kemp JA, Foster AC, Leeson PD, Priestley J, Tridgett R, Iversen LL, Woodruff GN (1988) 7-Chlorokynurenic acid is a selective antagonist at the glycine modulatory site of the $N$-methyl-D-aspartate receptor complex. Proc Natl Acad Sci USA 85:6547-6550.

Larson J, Lynch G (1986) Induction of synaptic potentiation in hippocampus by patterned stimulation involves two events. Science 232: 985-933.

Larson J, Wong D, Lynch G (1986) Patterned stimulation at the theta frequency is optimal for the induction of hippocampal long-term potentiation. Brain Res 368:347-350.

Larson J, Xiao P, Lynch G (1993) Reversal of LTP by theta frequency stimulation. Brain Res 600:97-102.

Linden DJ (1994) Long-term synaptic depression in the mammalian brain. Neuron 12:457-472.

Lisman J (1989) A mechanism for the Hebb and the anti-Hebb processes underlying learning and memory. Proc Natl Acad Sci USA 86:9574-9578.

Malenka RC, Nicoll RA (1993) NMDA-receptor-dependent synaptic plasticity: multiple forms and mechanisms. Trends Neurosci 16:521527.

Malinow R, Madison DV, Tsien RW (1988) Persistent protein kinase activity underlying long-term potentiation. Nature 335:820-824.

Matthies H Jr, Behnisch T, Kase H, Matthies H, Reymann KG (1991) Differential effects of protein kinase inhibitors on pre-established long-term potentiation in rat hippocampal neurons in vitro. Neurosci Lett 121:259-262.

Moore SD, Barr DS, Wilson WA (1993) Seizure-like activity disrupts LTP in vitro. Neurosci Lett 163:117-119.

Mulkeen D, Anwyl R, Rowan M (1988) The effects of external calcium on long-term potentiation in the rat hippocampal slice. Brain Res 447:234-238.

Mulkey RM, Malenka RC (1992) Mechanisms underlying induction of homosynaptic long-term depression in area $\mathrm{CA} 1$ of the hippocampus. Neuron 9:967-975.

Mulkey RM, Herron CE, Malenka RC (1993) An essential role for protein phosphatases in hippocampal long-term depression. Science 261:1051-1055.

O'Dell TJ, Kandel ER (1994) Low-frequency stimulation erases LTP through an NMDA receptor-mediated activation of protein phosphatases. Learn Memory 1:129-139.

Squire LR (1986) Memory functions as affected by electroconvulsive therapy. Ann NY Acad Sci 462:307-314.

Staubli U, Lynch G (1987) Stable hippocampal long-term potentiation elicited by theta pattern stimulation. Brain Res 435:227-234. 
Staubli U, Lynch G (1990) Stable depression of potentiated synaptic responses in the hippocampus with $1-5 \mathrm{~Hz}$ stimulation. Brain Res 513:113-118.

Stevens CF, Wang YW (1993) Reversal of long-term potentiation by inhibitors of haem oxygenase. Nature 364:147-149.

Stringer JL, Guyenet PG (1983) Elimination of long-term potentiation in the hippocampus by phencyclidine and ketamine. Brain Res 258: 159-164.

Teyler TJ, Cavus I, Coussens C, Discenna P, Grover L, Lee YP, Little
Z (1994) Multideterminant role of calcium in hippocampal synaptic plasticity. Hippocampus 4:623-634.

Thompson PJ (1991) Memory function in patients with epilepsy. Adv Neurol 55:369-384.

Wexler EM, Stanton PK (1993) Priming of homosynaptic long-term depression in hippocampus by previous synaptic activity. Neuroreport 4:591-594.

Yamamoto C, Sawada S (1981) Important factors in induction of longterm potentiation in thin hippocampal sections. Exp Neurol 74:122130 . 